\section{The potential benefits of a new poliovirus vaccine for long-term poliovirus risk management}

\author{
Radboud J Duintjer Tebbens* ${ }^{* 1} \&$ Kimberly M Thompson'
}

\begin{abstract}
Aim: To estimate the incremental net benefits (INBs) of a hypothetical ideal vaccine with all of the advantages and no disadvantages of existing oral and inactivated poliovirus vaccines compared with current vaccines available for future outbreak response. Methods: INB estimates based on expected costs and polio cases from an existing global model of long-term poliovirus risk management. Results: Excluding the development costs, an ideal poliovirus vaccine could offer expected INBs of US\$1.6 billion. The ideal vaccine yields small benefits in most realizations of long-term risks, but great benefits in low-probability-highconsequence realizations. Conclusion: New poliovirus vaccines may offer valuable insurance against long-term poliovirus risks and new vaccine development efforts should continue as the world gathers more evidence about polio endgame risks.
\end{abstract}

First draft submitted: 24 June 2016; Accepted for publication: 22 August 2016,

Published online: 10 November 2016

Widespread poliovirus vaccination started in 1955 with the introduction of the injectable inactivated poliovirus vaccine (IPV) [1]. Despite impressive reductions in polio incidence from wild polioviruses (WPVs) $[2,3]$, outbreaks continued due to suboptimal seroconversion and failure of IPV to prevent fecal-oral transmission $[4,5,6,7]$. In the early 1960 s, the live, attenuated oral poliovirus vaccine (OPV) became available, offering much better intestinal immunity than IPV [7,8,9], much cheaper production, ease of oral administration and the beneficial ability to infect and immunize close contacts of vaccine recipients $[10,11]$. These properties favored OPV as a tool to rapidly interrupt transmission through campaigns that vaccinated many individuals in a short period of time [8,12].

Despite its benefits, OPV comes with disadvantages. First, despite its attenuation, OPV virus causes vaccine-associated paralytic poliomyelitis (VAPP) in approximately one per million susceptible OPV recipients (compared with 1/200-1/2000 for the three WPV serotypes) [13]. Second, replication of the OPV virus in populations with low coverage can result in reversion of the attenuating mutations, which increases the neurovirulence and fitness of the virus and can lead to outbreaks of circulating vaccine-derived poliovirus (cVDPV) [10,14-18]. Third, long-term replication of the OPV virus can also occur in individuals with B-cell-related primary immunodeficiency diseases (PIDs), as reported in more than 70 individuals infected for over 6 months with immunodeficiencyassociated vaccine-derived poliovirus (iVDPV) [16], although even among PID patients long-term poliovirus excretion remains rare $[19,20]$. None of the identified iVDPVs triggered polio outbreaks to date, but this could merely reflect the currently high levels of population immunity to transmission in the communities in which PID patients tend to survive long enough to develop longterm iVDPV infections (i.e., typically in settings with little fecal-oral transmission, such that the effect of IPV on oropharyngeal transmission can effectively prevent widespread transmission) [20].

'Kid Risk, Inc., 10524 Moss Park Rd., Ste. 204-364, Orlando, FL 32832, USA

*Author for correspondence: rdt@kidrisk.org

\section{KEYWORDS}

- dynamic modeling

- eradication $\bullet$ OPV

cessation $\bullet$ polio $\bullet$ risk

management

- vaccine development 
While direct estimation of transmissibility and human neurovirulence remains challenging, no epidemiologic or laboratory data exist to suggest that VDPVs (i.e., cVDPVs and iVDPVs) behave differently than homotypic WPVs in terms of their ability to transmit in populations and cause paralysis [15,16,21]. Finally, while OPV provided good seroconversion in high-income settings, seroconversion rates remains lower in developing country settings [22], possibly due to interference with other enteroviruses [23], which means that effective OPV immunization requires multiple doses to protect individuals, particularly in low-hygiene settings [24].

Despite the risks and relatively low OPV seroconversion rates, since the World Health Assembly resolved in 1988 to globally eradicate polio [25], the Global Polio Eradication Initiative (GPEI) relied almost exclusively on OPV to successfully interrupt WPV transmission in the most challenging environments. Naturally occurring serotype 2 WPV and serotype 3 WPV (WPV3) did not cause any detected paralytic poliomyelitis cases since 1999 [26] and 2012 [27], respectively, and indigenous serotype 1 WPV (WPV1) transmission continues only in three remaining endemic countries as of 2016 (i.e., Pakistan, Afghanistan and Nigeria) [28]. Recognition of the risks of OPV as unacceptable after global WPV eradication [29] motivated a World Health Assembly resolution to globally coordinate OPV cessation after certification of eradication [30]. Coordinated cessation of serotype 2-containing OPV (OPV2 cessation) occurred in April and May 2016 [31], with cessation of the remaining two OPV serotypes (OPV13 cessation) planned for after certification of global WPV1 and WPV3 eradication $[32,33]$. Many relatively higher income countries switched from OPV to IPV for their national immunization programs to eliminate VAPP after they became polio-free [34,35], and the GPEI currently recommends at least one IPV dose in all countries to provide some protection against serotype 2 after OPV2 cessation for vaccine recipients [31], with reduction in VAPP occurring when infants receive IPV before receiving OPV [13]. Current IPV vaccines exhibit enhanced potency compared with the original IPV formulation from the 1950s and provide high seroconversion in all settings [1]. The GPEI further coordinated efforts to make IPV more affordable and improve delivery strategies [36]. However, an episode of widespread asymptomatic WPV1 transmission occurred in Israel in 2013-2014 despite very high IPV coverage, which confirmed that despite its enhanced potency, using IPV only does not provide sufficient protection from participation in (fecal-oral) poliovirus transmission even in a subtropical environment with probably above-average hygiene and sanitation [37,38].

Numerous recent articles discuss the complex policy choices following global polio eradication [34,35,39-50]. Several modeling studies quantified the issues and trade-offs associated with vaccine choices for the polio endgame and suggest that OPV intensification prior to OPV cessation represents the best way to minimize the risk of cVDPV outbreaks following OPV cessation [20,29,33,51-54]. The model assumptions proved consistent [55,56] with evidence from clinical trials that subsequently demonstrated a greater boosting effect of IPV than OPV in individuals with immunity from a previous live poliovirus infection [57,58]. The model demonstrates limited effect of IPV on population immunity to transmission in settings conducive to fecal-oral transmission, because transmission dynamics depend primarily on fully susceptible individuals and providing IPV in addition to OPV to children captured by immunization efforts yields a small incremental benefit and consequently little effect of IPV use in areas at highest risk of cVDPVs after OPV cessation [53]. An integrated global heath-economic model of long-term poliovirus risk management policies (i.e., the global model) found that iVDPV or other poliovirus reintroductions occurring in settings conducive to fecal-oral transmission and beyond a 5-year window after OPV cessation (until which the model assumed OPV can be used safely for outbreak response) could result in uncontrolled outbreaks in some cases [33]. While over $90 \%$ of model realizations did not result in uncontrolled outbreaks, the ones that did would force most countries to resume routine OPV use because outbreak response with IPV could not stop transmission. Further analyses of outbreak response strategies suggested little ability to improve outbreak control with the currently available vaccines, unless the world remained willing to accept an increasing risk of using OPV for outbreak response at a time when most of the world would not maintain sufficient population immunity to prevent transmission of OPV-related viruses [54]. The reality of cVDPVs, iVDPVs, potential breaches in containment from laboratories or IPV production sites and potential intentional reintroductions [51,59] 
motivate the GPEI to develop and implement risk management strategies, because as long as polioviruses exist, complete prevention of poliovirus reintroductions after OPV cessation cannot be guaranteed.

OPV cessation will affect the ability to use OPV for research. This means that testing of new interventions (e.g., poliovirus antiviral compounds, new poliovirus vaccines) that require OPV use for demonstrating effectiveness or noninferiority must occur prior to OPV cessation. Recognizing this limited time window and the potential need for better poliovirus vaccines in the future, the GPEI and others continue to consider the possibility of developing new vaccines that combine the advantages of OPV and IPV [35,50,60-69]. However, no existing studies provide quantitative estimates of the potential impact of new poliovirus vaccines to inform decisions related to further investments into new poliovirus vaccine development. This study aims to provide health and economic context by using an existing global model [33] to assess the potential benefits of a hypothetical new poliovirus vaccine with all the advantages but none of the disadvantages of OPV and IPV (i.e., ideal poliovirus vaccine).

\section{Methods}

Our analysis follows guidelines for health-economic analyses [70,71] to estimate the incremental net benefits (INBs) over a 40-year period of using either the existing poliovirus vaccines or an ideal new poliovirus vaccine for future outbreak response compared with the status quo at the time of the 2013-2018 GPEI Strategic Plan [32]. The global model [33] integrates a dynamic poliovirus transmission and OPV evolution model [72] that characterizes poliovirus prevalence (including cVDPVs) and population immunity to transmission with randomly occurring poliovirus reintroductions (i.e., OPV not withdrawn from the field, iVDPVs, releases of seed strains from vaccine manufacturing sites and/or intentional and unintentional other releases from laboratories) [20], economic implications of vaccination and polio cases [34,73], and variability and mixing among 710 global subpopulations (with approximately 10 million people each in 2013) (see Supplementary Material). The global model characterizes variability between these subpopulations in costs by World Bank income level [74], poliovirus vaccine use, surveillance, and conditions related to poliovirus transmission, including the inherent poliovirus transmissibility using the basic reproduction number $\left(\mathrm{R}_{0}\right)$ of WPV1 (with $\mathrm{R}_{0}$ values for other serotypes and OPV-related viruses linked to the WPV1 $R_{0}$ using fixed relative $R_{0}$ values). One stochastic iteration of the global model (i.e., a possible realization of the future) produces estimated costs and cases between 2013 and 2052 associated with each considered course of action (i.e. policy, defined as set of choices about vaccination, outbreak response and stockpiles, polio antiviral drug use, and surveillance). Averaging over multiple stochastic iterations yields an estimate of the expected INBs of a prospective policy compared with the status quo policy of continued OPV use (i.e., the reference case). The INBs represent the difference of the expected economic value of averted polioassociated deaths and disability and the expected costs for the policy compared with the status quo. As mentioned, the currently available vaccines do not control some outbreaks in some stochastic realizations [33,54], which ultimately leads to spread to all populations that cannot sustain high enough population immunity to transmission without OPV to prevent imported viruses from establishing transmission. In these cases, the failure of outbreak response efforts leads to a need to restart OPV use in those populations. In the global model, we operationalize this by assuming that if the cumulative number of polio cases since 2016 exceeds a cut-off of 50,000, then all countries revert to their 2013 routine immunization (RI) schedule from the first day of the year after they exceed this threshold until the end of the analytical time horizon (i.e., OPV restart). During that time period, the model assumes for the base case that these countries incur the costs and polio cases associated with never stopping routine OPV use. The 50,000 case threshold for OPV restart effectively separates model runs in which outbreak response activities control all outbreaks from runs in which outbreaks go out of control, and the results remain insensitive to the precise cut-off value within a range of 15,000 50,000 cumulative polio cases, with larger cut-off values unlikely to occur without a decision to restart OPV [33]. Prior analyses using the global model considered OPV restarts with or without resumption of regular preventive supplemental immunization activities (pSIAs) with OPV according to the schedule in the global model for polio-free countries in 2013 [33,75], with no outbreak response SIAs (oSIAs) for either possibility. These analyses found a relatively small 
difference between the two cases given that the higher costs for OPV restart with pSIAs offsets the higher costs associated with polio cases for OPV restart without pSIAs [33].

To explore the health and economic benefits of an ideal poliovirus vaccine, we focus on the global model baseline policy that reflects the GPEI Strategic Plan 2013-2018 of WPV eradication, OPV2 cessation in 2016 after meeting several prerequisites (including IPV introduction in all countries, establishment of an mOPV2 stockpile and outbreak response guidelines) and OPV13 cessation after certification of WPV eradication [32]. Specifically, the baseline policy assumes introduction of at least one IPV dose in RI in all countries starting before OPV2 cessation and continuing until 5 years after global OPV13 cessation [33]. At that point, low and lower middle-income countries stop using IPV in RI while upper middle- and high-income countries continue to use IPV-only for RI. Populations in all income levels conduct oSIAs in the event that any reintroduced polioviruses cause new (detected) paralytic cases. In the baseline policy, oSIAs use monovalent OPV of the detected poliovirus serotype during the first 5 years after homotypic OPV cessation, and IPV thereafter. We consider an ideal poliovirus vaccine scenario that assumes availability of a new and hypothetical poliovirus vaccine for all oSIAs from 5 years after homotypic OPV cessation. For the ideal poliovirus vaccine scenario, we assume that any OPV restarts use the new vaccine and resume regular vaccination only for the serotype for which the uncontrolled outbreaks occurred. We considered restarts with the ideal poliovirus vaccine either without any SIAs other than oSIAs already planned before the cut-off was reached, or restarts that continue oSIAs as long as outbreaks continue without resumption of pSIAs. In the context of modeling the ideal poliovirus vaccine, these represent more beneficial OPV restart options than the assumed resumption of OPV use of all serotypes used as of 2013 for the base case scenario. Unless otherwise noticed, we report results for OPV restarts that do not resume pSIAs (baseline policy) and do not continue oSIAs (ideal poliovirus vaccine scenario).

Table 1 lists the attributes we assumed for the ideal poliovirus vaccine scenario, reflecting an ideal vaccine that combines all of the advantages of IPV or OPV (whichever performs better for the attribute in Table 1) without their drawbacks. The attributes include cost-related model inputs (price, administration costs, wastage), model inputs affecting the impact of the ideal poliovirus vaccine on individual immunity to polio (i.e., take rate) and population immunity to poliovirus transmission (i.e., contribution to oropharyngeal and fecal-oral transmission), and model inputs affecting the risks (i.e., VAPP rates, reversion times, longterm infection ability, production site releases). The ideal poliovirus vaccine assumptions imply that the vaccine provides secondary immunity to contacts of vaccine recipients similar to OPV, but that the vaccine virus does not cause VAPP and cannot revert to a more neurovirulent and transmissible phenotype. Although in theory an ideal poliovirus vaccine could offer an improvement over both IPV and OPV in some individual attributes (e.g., lower price than OPV, better take rates than IPV, less waning and/or contribution to transmission than OPV), this appears very unlikely in practice for most attributes. Therefore we assume that the ideal poliovirus vaccine would not perform better than the best for IPV and OPV for any individual attribute. We did not consider vaccine attributes that affect the ability to stockpile the vaccine (e.g., volume and shelf life of the filled product) or the cold chain requirements (e.g., thermostability).

Following our economic analysis of the global model with 100 stochastic iterations [33], we performed an additional 1000 iterations to better estimate the probabilities and consequences of rare events, including OPV restarts [76,77]. Rare events that ultimately triggered OPV restarts involved iVDPV introductions (either in places with very high poliovirus transmissibility, or in places with moderate poliovirus transmissibility but long after OPV cessation) and intentional or unintentional releases from laboratories [76]. Consistent with the more recent studies, we perform the analyses in this study based on a stratified set of 120 iterations that includes all 57 OPV restart iterations (i.e., iterations in which an OPV restart occurred in the base case) from the full set of 1000 iterations as well as 63 representative iterations with no OPV restart in the base case, and we use weighted averages to estimate expected outcomes [77]. This approach allows us to appropriately account for the impact of the ideal poliovirus vaccine 
based on the probabilities of favorable (i.e., all outbreaks controllable with existing vaccines) or nonfavorable (i.e., uncontrolled outbreaks with use of the existing vaccines only) iterations. We report the number of OPV restarts, expected outbreak response doses and costs, expected total costs, expected polio cases and expected INBs of the ideal poliovirus vaccine scenario compared with the global baseline policy without the ideal poliovirus vaccine. We adopt the $3 \%$ discount rate from the global model and express all monetary outcomes in year 2013 US \$ [33]. We also highlight specific results from individual iterations to illustrate the possible effects of an ideal new vaccine on the behavior of potential future outbreaks.

\section{Results}

Table 2 summarizes the impact of an ideal poliovirus vaccine on major outcomes of the global model. The ideal poliovirus vaccine offers the OPV benefit of inducing rapid and substantial increases in population immunity to transmission following deployment in response to outbreaks without the OPV risk of seeding new cVDPV outbreaks elsewhere or creating new long-term iVDPV excretors [54]. This allows control of most outbreaks even if they occur long after OPV cessation and/or in populations with high $\mathrm{R}_{0}$ values reflecting conditions that favor intense fecal-oral poliovirus transmission. Consequently, we find that 54 of 57 OPV restart iterations with the base case do not result in an OPV restart if an ideal poliovirus vaccine were available for oSIAs from 5 years after homotypic OPV cessation, corresponding to a $95 \%$ reduction in the probability of OPV restarts. The three remaining OPV restart iterations all resulted from inadvertent or intentional release of poliovirus from containment failures into populations with high $\mathrm{R}_{0}$ values and thus did not reflect risks associated with OPV use or IPV production. Controlling outbreaks that trigger oSIAs beyond 5 years after homotypic OPV cessation required on average approximately 40 million doses of the ideal poliovirus vaccine. However, it prevented repeated and often ultimately unsuccessful oSIAs with IPV, and consequently resulted in an overall reduction in the average number of total oSIA doses used from over 900 million for the base case to less than 200 million with the ideal poliovirus vaccine. Some mOPV use still occurs with the ideal poliovirus vaccine because we assume that it becomes available only 5 years after homotypic OPV cessation (Table 1).

Table 2 shows that the most important reduction in expected polio cases comes from the prevention of OPV restarts, with a small effect on the much more common iterations that did not lead to OPV restarts for the base case, because these typically do not require many oSIAs beyond 5 years after homotypic OPV cessation. Notably, with the ideal poliovirus vaccine, we found that for two of the three OPV restarts that still occur, the ideal poliovirus vaccine eventually stops all transmission of the re-established outbreak virus after reaching the OPV restart cut-off, such that cessation of the ideal poliovirus vaccine could also occur (although we did not model this option). The overall reduction in expected polio cases (i.e., weighted by the probability of OPV restarts in the base case) equals almost 60,000 (96\%). The reduction in expected vaccination costs remains moderate and equals US $\$ 1.1$ billion (3\%) because the continued routine use of IPV in higher income countries and expected time-limited use of IPV in lower income populations contribute significantly to the costs independent of the availability of an ideal poliovirus vaccine [77]. The combined reduction in expected costs and cases translates into an increase in the expected INBs of US $\$ 1.6$ billion (10-13\% increase, depending on comparison to a reference case that assumes continued SIAs or not) associated with the development of an ideal poliovirus vaccine for outbreak response.

Figure 1 gives three examples from different global model iterations of the behavior of the ideal poliovirus vaccine compared with IPV in response to poliovirus reintroductions that occur more than 5 years after homotypic OPV cessation. In Figure 1A, a serotype 3 iVDPV introduction from a chronic iVDPV excretor occurs over 8 years after OPV13 cessation. This type of introduction remains most likely in a population with relatively lower $R_{0}$ values for polio because of the longer expected survival of immunodeficient patients in this type of setting compared with higher $\mathrm{R}_{0}$ settings [20]. The lower $R_{0}$ values imply more ability of IPV to stop poliovirus outbreaks, and indeed for this outbreak in a population with a WPV1 $\mathrm{R}_{0}$ of 7 (i.e., WPV3 $R_{0}$ of 5.25), aggressive outbreak response with two series of four IPV oSIAs 
RESEARCH ARTICLE Duintjer Tebbens \& Thompson

Table 1. Assumed attributes of oral poliovirus vaccine, inactivated poliovirus vaccine and an ideal new poliovirus vaccine.

\begin{tabular}{|c|c|c|c|}
\hline Vaccine attribute (unit) & Assumption for OPV & Assumption for IPV & $\begin{array}{l}\text { Assumption for hypothetical } \\
\text { ideal poliovirus vaccine }\end{array}$ \\
\hline Time of availability for oSIAs & $\begin{array}{l}\text { Until } 5 \text { years after homotypic } \\
\text { OPV cessation }\end{array}$ & $\begin{array}{l}\text { From } 5 \text { years after homotypic } \\
\text { OPV cessation }{ }^{+}\end{array}$ & $\begin{array}{l}\text { From } 5 \text { years after homotypic } \\
\text { OPV cessation }\end{array}$ \\
\hline \multicolumn{4}{|l|}{ Price per dose ${ }^{\ddagger}$ (US\$/dose): } \\
\hline - Low-income country & 0.12 & 1.30 & 0.12 \\
\hline - Lower middle-income country & 0.12 & 2.30 & 0.12 \\
\hline - Upper middle-income country & 0.13 & 3.20 & 0.13 \\
\hline - High-income country & 0.16 & 13.00 & 0.16 \\
\hline Effective vaccine wastage in SIAs & $44 \%$ & $44 \%$ & $44 \%$ \\
\hline \multirow{4}{*}{$\begin{array}{l}\text { Average per-dose take rates: } \\
\text { - Low or lower middle-income country } \\
\text { - Upper middle income country } \\
\text { - High-income country }\end{array}$} & \multirow{4}{*}{$\begin{array}{l}\text { Varies by population and OPV } \\
\text { formulation }\end{array}$} & & mOPV take rates \\
\hline & & 0.63 & \\
\hline & & 0.70 & \\
\hline & & 0.75 & \\
\hline $\begin{array}{l}\text { Contribution to fecal-oral and } \\
\text { oropharyngeal transmission }\end{array}$ & $\begin{array}{l}\text { Varies by number of live } \\
\text { poliovirus infections and } \\
\text { waning stage }^{\S}\end{array}$ & $\begin{array}{l}\text { Varies by number of successful } \\
\text { IPV vaccinations and waning } \\
\text { stage }^{\S}\end{array}$ & Same as OPV \\
\hline & 0 & 0 \\
\hline - Serotype 1 & $0.26 \times 10^{-6}$ & & \\
\hline - Serotype 2 & $1.2 \times 10^{-6}$ & & \\
\hline & $1.8 \times 10^{-6}$ & & \\
\hline & No reversion & No reversion \\
\hline - Serotypes 1 and 3 & 620.5 & & \\
\hline - Serotype 2 & 408 & & \\
\hline \multicolumn{2}{|l|}{ Relative $\mathrm{R}_{0}$ of OPV parent strain vs } & 0 & \\
\hline homotypic WPV or cVDPV:" & 0.37 & & 0.37 \\
\hline - Serotype 1 & 0.55 & & 0.55 \\
\hline - Serotype 2 & 0.25 & & 0.25 \\
\hline \multicolumn{4}{|l|}{ - Serotype 3} \\
\hline $\begin{array}{l}\text { Fraction of primary immunodeficiency } \\
\text { disease patients that may develop a } \\
\text { long-term infection (if infected and } \\
\text { surviving) }\end{array}$ & $\begin{array}{l}0.01 \text { (prolonged) or } 0.005 \\
\text { (chronic, CVID patients only) }\end{array}$ & 0 & 0 \\
\hline Rate of release of seed strains (1/year) & N/A (no ongoing production) & $1 / 5^{\#}$ & 0 \\
\hline \multicolumn{4}{|c|}{$\begin{array}{l}\text { PPopulations that already use IPV-only in } 2013 \text { use IPV for oSIAs throughout. } \\
\text { FFor IPV, price per full dose assumed, and the use of fractional doses would reduce the antigen price accordingly. } \\
\text { sNumerical values summarized in Duintjer Tebbens et al. [54]. } \\
\text { "WPV and cVDPV Ro values for serotypes } 2 \text { and } 3 \text { assumed equal to } 0.9 \text { and } 0.75 \text { times those for serotype } 1 \text {, respectively. } \\
\text { "Nature of seed strain depends on location, with high-income countries assumed to use wild poliovirus seed strains and manufacturing in other settings assume to switch to } \\
\text { Sabin seed strains [33]. } \\
\text { cVDPV: Circulating vaccine-derived poliovirus; CVID: Common variable immunodeficiency disease; IPV: Inactivated poliovirus vaccine; mOPV: Monovalent OPV; OPV: Oral } \\
\text { poliovirus vaccine; OSIA: Outbreak response SIA; R: Basic reproduction number; RC: Reference case; SIA: Supplemental immunization activity; VAPP: Vaccine-associated paralytic } \\
\text { poliomyelitis; US\$: Year } 2013 \text { US\$; WPV: Wild poliovirus. } \\
\text { Assumptions for OPV and IPV based on prior work [20,33,72,80]. }\end{array}$} \\
\hline
\end{tabular}

eventually controls the outbreak after incurring approximately 80 polio cases. Use of an ideal poliovirus vaccine would control the outbreak after the first series of four oSIAs and limit the number of polio cases to approximately 40. In this example, no spread occurred beyond the subpopulation that experienced the introduction for the base case or ideal poliovirus vaccine scenario. The type of behavior in Figure 1A represents the most common type of behavior associated with outbreaks that occur more than 5 years after homotypic OPV cessation.
Figure 1B provides an example of an outbreak caused by a serotype 2 iVDPV excretor in which use of an ideal poliovirus vaccine prevents an OPV restart. For the base case, aggressive IPV oSIAs help limit the outbreak for three series of four rounds, but do not stop all transmission. Eventually, continued transmission leads to exportations of the outbreak virus to neighboring subpopulations, triggering new outbreaks and IPV oSIAs, which ultimately results in over 50,000 polio cases and an OPV restart. In contrast, oSIAs with the ideal 
poliovirus vaccine successfully stop the outbreak after the first series of four rounds. The type of behavior in Figure 1B occurs much less frequently (i.e., approximately $5 \%$ of iterations) compared with the behavior demonstrated by Figure $1 \mathrm{~A}$, but it remains typical for iterations with OPV restarts for the base case.

Figure $1 \mathrm{C}$ shows a very rare example (i.e., representative of $0.3 \%$ of all iterations) in which the ideal poliovirus vaccine did not prevent the OPV restart, which occurs in 2042 (not shown), caused by an (un)intentional release of WPV1 from a laboratory. However, in this example, the ideal poliovirus vaccine prevents over 900,000 polio cases during 2040-2041, limits the propagation of the outbreak and delays the OPV restart by 1 year compared with the base case.

\section{Discussion}

New poliovirus vaccines may offer valuable insurance against long-term poliovirus risks and new vaccine development efforts should continue at least until the world gathers more evidence about the probability and consequences of live poliovirus reintroductions during OPV cessation and the polio endgame. Developing a new vaccine will require separate investments for all three serotypes, with the possibility of the development of monovalent or combined vaccine formulations for future outbreak response. The ultimate benefits of the new vaccine will need to subtract the actual investments required to fully develop the new vaccines and make them available for use in outbreak response and consider any attributes of the vaccine that make it less than ideal. The total expected INB of US\$1.6 billion for a hypothetical ideal poliovirus vaccine provides a potential upper bound of the benefits for all three serotypes, which if equally divided would economically support investments of up to approximately US\$500 million for each serotype. For context, estimated costs to develop a new vaccine range from US\$500 million to US $\$ 1$ billion, depending on the complexity of the vaccine and including construction of manufacturing facilities [78]. However, a new poliovirus vaccine for emergency use would represent a modification of already existing vaccines and may potentially bypass some of the production challenges and/or regulatory requirements, and thus uncertainty remains about the costs to make a new poliovirus vaccine available. Although serotype $2 \mathrm{OPV}$ strains remain the closest to typical homotypic WPVs in terms of their phenotypic properties and they evolve the fastest to

Table 2. Global model results with or without the ideal poliovirus vaccine based on a stratified set of 120 stochastic iterations [77], representing 1000 iterations of the global model [33].

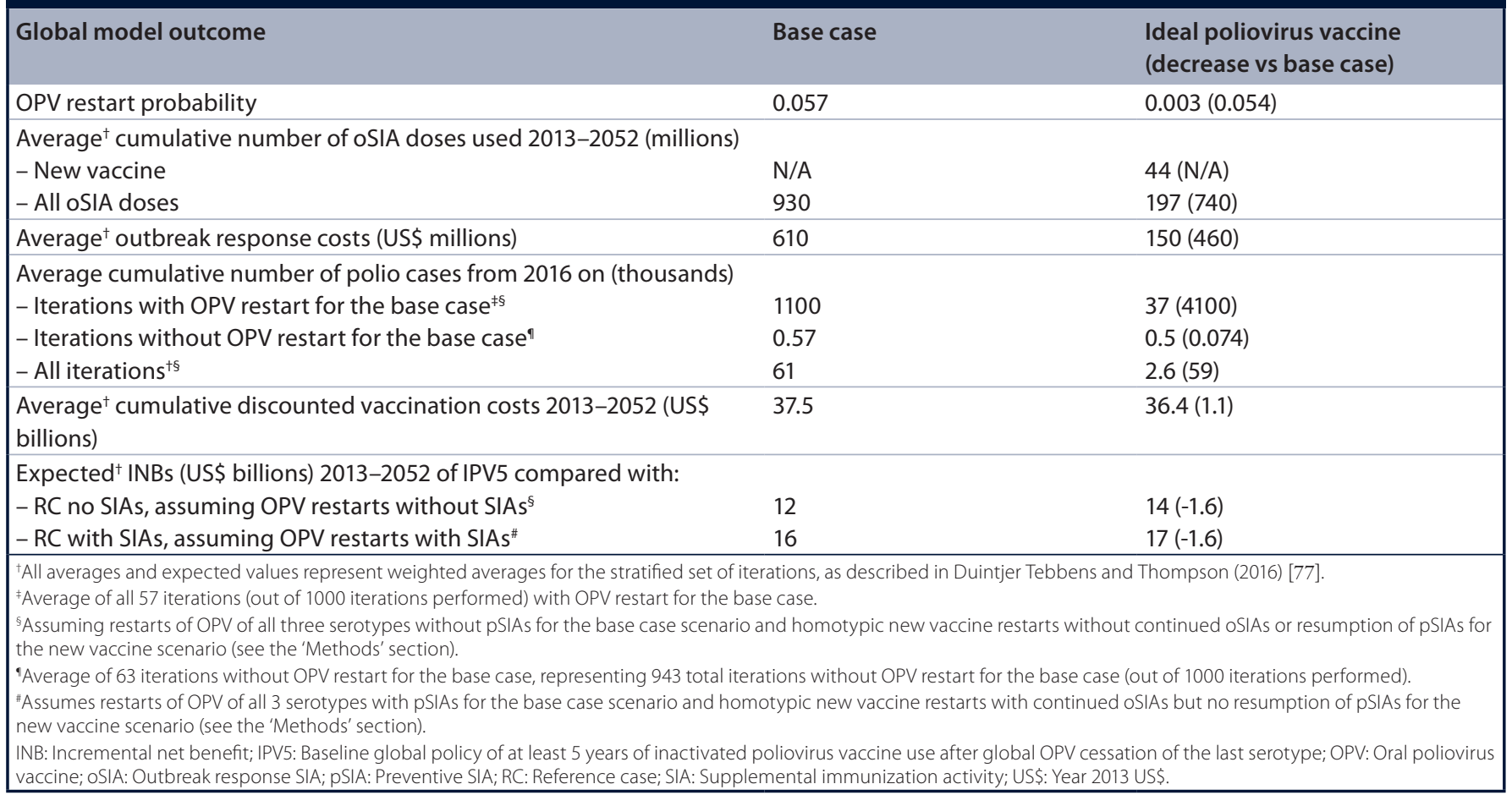


RESEARCH ARTICLE Duintjer Tebbens \& Thompson

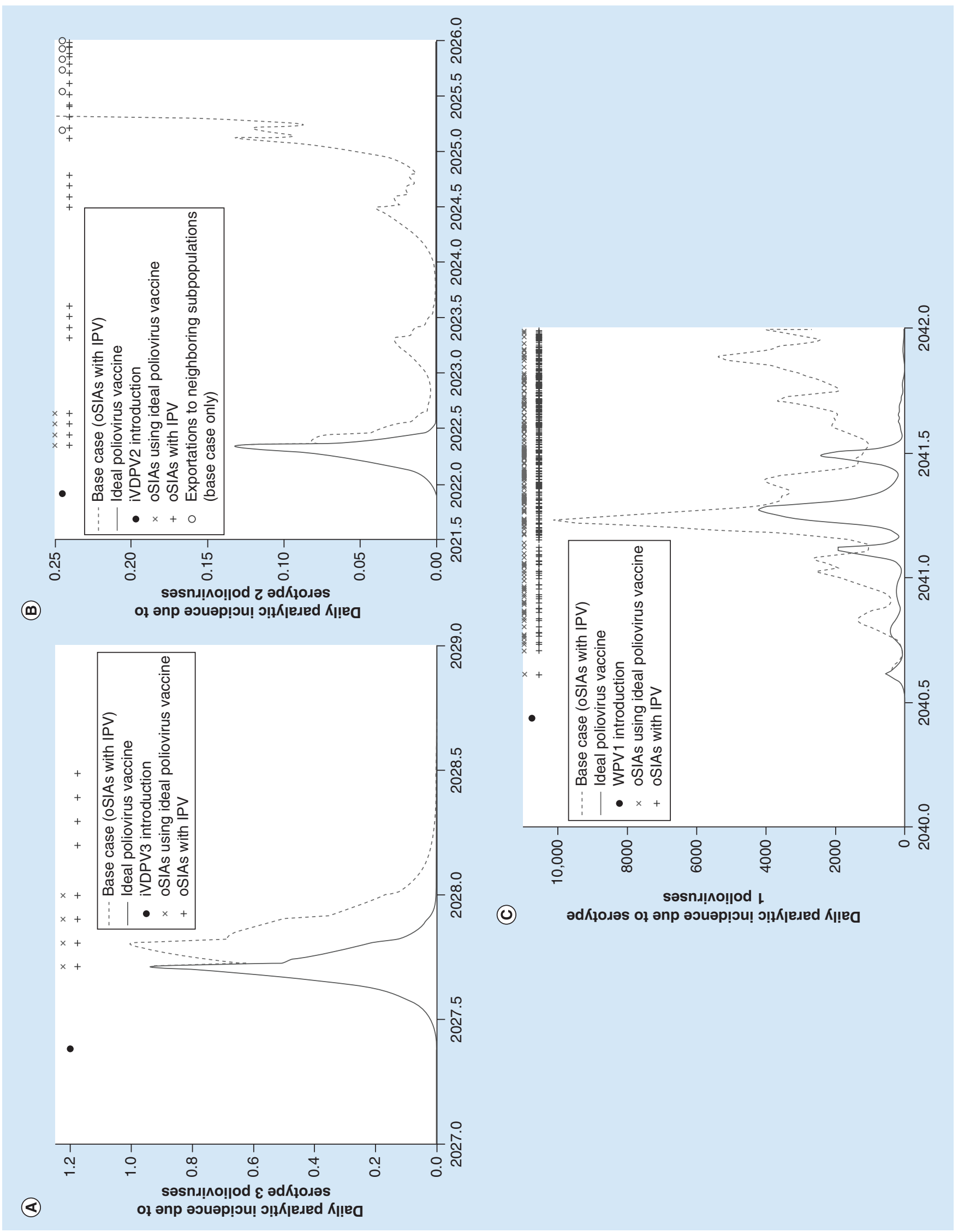


Figure 1. Three examples of different types of behaviors observed for use of an ideal poliovirus vaccine instead of the existing inactivated poliovirus vaccine in oSIAs. (A) Substantial reduction in consequences of an iVDPV3 outbreak that aggressive oSIAs with IPV also ultimately control. (B) Substantial reduction in consequences of an iVDPV2 outbreak that would ultimately lead to an OPV restart even with aggressive IPV oSIAs, but not with oSIAs using the new vaccine. (C) Reduction in consequences and propagation of a WPV1 outbreak caused by an (un)intentional release from a laboratory, but insufficient impact of the new vaccine to prevent an OPV restart. IPV: Inactivated poliovirus vaccine; OPV: Oral poliovirus vaccine; iVDPV2: Serotype 2 immunodeficiency-associated vaccine-derived poliovirus; iVDPV3: Serotype 3 immunodeficiencyassociated vaccine-derived poliovirus; oSIA: Supplemental immunization activity; WPV1: Serotype 1 wild poliovirus.

VDPVs [15], wild and fully reverted serotype 1 polioviruses probably remain the most transmissible and neurovirulent [72]. Consequently, we observed the highest proportion of OPV restarts associated with serotype 1 events (67\% of OPV restarts), followed by serotype $2(25 \%)$ and very few serotype 3 (9\%) [76]. While development of new serotype 2 poliovirus vaccines represents the highest current priority given that OPV2 cessation already occurred, we expect the greatest health-economic benefits associated with new serotype 1 poliovirus vaccines.

The models we used for this analysis come with limitations related to uncertainties and our choice of a deterministic framework for transmission within populations, as previously discussed $[33,72,79]$. Particularly, uncertainties about the kinetics of outbreak and vaccine virus spread between populations that affect the expected consequences of rare poliovirus reintroductions and the ability of different vaccines to control outbreaks or seed new outbreaks. As we learn more about the risks of cVDPVs and iVDPVs after OPV cessation and the potential benefits and uses of poliovirus antiviral drugs currently under development, further analyses may suggest potentially higher or lower expected INBs of new poliovirus vaccines [76]. The estimated INBs will also depend on the timing of the availability of the new vaccine, with our assumptions about the ideal vaccine becoming globally available 5 years after OPV13 cessation potentially representing an optimistic assumption. The timing of when new poliovirus vaccines become available for use in outbreak response will depend on investments in these vaccines going forward. The number of paralytic cases that would occur with use of the existing poliovirus vaccine will further depend on the actual impact of IPV on transmission in different settings and on the proportion of vaccine recipients protected from poliomyelitis disease as potentially more immunogenic IPV vaccines become available [69]. As new vaccine developments proceed, further analysis may explore more realistic timing and research and development cost estimates. In the absence of a fully characterized new poliovirus vaccine, our analysis focused on providing an upper bound of the expected INBs for a new vaccine with ideal properties. Further analyses that relax the ideal properties may provide context related to more realistic new vaccines that could include more of the disadvantages of OPV (e.g., less than $100 \%$ stable virus with non-zero chance of VAPP or reversion) or IPV (e.g., absence of secondary immunization of contacts, higher antigen or delivery costs), or possibly even further improvements (e.g., lower wastage associated with single vials or greater thermostability, fewer cold chain requirements, or better ability to stockpile). The consequences of uncontrolled outbreaks and the specifics of when and how the world would restart OPV remain unknown, but significantly affect the expected health and economic estimates. Further analyses and deliberations should consider the true implications of unlikely but possible OPV restarts.

\section{Future perspective}

With global OPV2 cessation, the world started its phased transition to the post WPV-eradication era, which will result in unprecedented low levels of population immunity to poliovirus transmission. Already, the isolation of a serotype 2 VDPV from sewage in Nigeria linked to persistent cVDPV2 circulation triggered the first deployment of mOPV2 from the stockpile [28]. Within the next few years, the world will observe whether and how rapidly OPV2 viruses die out, whether the mOPV2 outbreak response strategy can stop the outbreak virus, how much transmission of the mOPV2 virus occurs outside of the target population, what role IPV might play in outbreak response, whether iVDPV excretors in 
developing countries can start outbreaks once population immunity declines, and by extension, how well IPV can maintain global population immunity to transmission. We anticipate continued research into new poliovirus vaccines as the post-OPV2 cessation risks play out. However, uncertainty about long-term risks will persist and new poliovirus vaccine development may reach a point at which the costs of further development and regulatory requirements will become a major driver of decisions to continue. In the event that all candidate new poliovirus vaccines will require significantly more resources or continue to involve important risks of reversion to wild-type properties, then estimates of long-term risk based on the experience with OPV2 cessation will drive further investment decisions. Given the important health and economic stakes involved, collecting as much data as possible remains essential, particularly related to new poliovirus vaccine candidate properties, expected development costs, and the ability of the existing OPV and IPV to control outbreaks and successfully navigate the endgame.
Financial \& competing interests disclosure This work was supported by the Bill and Melinda Gates Foundation [OPP1129391]. The authors thank the Bill and Melinda Gates Foundation for financial support, but the contents of this manuscript are solely the responsibility of the authors. The authors have no other relevant affiliations or financial involvement with any organization or entity with a financial interest in or financial conflict with the subject matter or materials discussed in the manuscript apart from those disclosed.

No writing assistance was utilized in the production of this manuscript.

\section{Supplementary data}

To view the supplementary data that accompany this paper, please visit the journal website at: www.futuremedicine. com/doi/full/10.2217/fmb-2016-0126

\section{Open access}

This work is licensed under the AttributionNonCommercial-NoDerivatives 4.0 Unported License. To view a copy of this license, visit http://creativecommons.org/ licenses/by-nc-nd/4.0/

\section{EXECUTIVE SUMMARY}

\section{Limitations of oral poliovirus vaccine \& inactivated poliovirus vaccine for outbreak response}

- Inactivated poliovirus vaccine may not effectively control outbreaks long after oral poliovirus vaccine (OPV) cessation in settings conducive to fecal-oral transmission.

- As population immunity to transmission declines after OPV cessation, responding to an outbreak caused by a reintroduced poliovirus with OPV comes with a risk of creating new immunodeficiency-associated vaccine-derived polioviruses or circulating vaccine-derived polioviruses, but controlling the outbreak virus remains critical to protect the achievement of a polio-free world.

\section{Urgency of new poliovirus vaccine development}

- The completion of global cessation of serotype 2 OPV makes testing any new live poliovirus vaccines of this serotype increasingly difficult while also increasing the potential need for a new poliovirus vaccine to respond to outbreaks.

\section{Potential benefits of an ideal new poliovirus vaccine}

- An ideal poliovirus vaccine available for outbreak response 5 years after homotypic OPV cessation may offer expected incremental net benefits of up to approximately US\$1.6 billion, excluding development costs of the new vaccine.

- These benefits come primarily from preventing uncontrolled outbreaks and OPV restarts in a small fraction of model runs, with much less benefit in runs without uncontrolled outbreaks.

- The availability of an ideal new poliovirus vaccine would reduce the probability of an OPV restart by $95 \%$ and significantly reduce the amount or vaccine used for outbreak response.

- The benefits of existing new poliovirus vaccines candidates will depend on their actual properties and the costs needed for further development and licensing to make them available for use in outbreak response.

\section{Conclusion}

- New poliovirus vaccines may offer valuable insurance against long-term poliovirus risks.

- New vaccine development efforts should continue at least until the world gathers more evidence about the probability and consequences of live poliovirus reintroductions during the polio endgame. 


\section{References}

Papers of special note have been highlighted as:

- of interest $\bullet$ of considerable interest

1 Vidor E, Plotkin SA. Poliovirus vaccine - Inactivated. In: Vaccines. Plotkin SA, Orenstein WA, Offit PA (Eds). Saunders Elsevier, PA, USA, 573-597 (2013).

2 Stickle G. Observed and expected poliomyelitis in the United States, 1958-1961. Am. J. Public. Health. 54, 1222-1229 (1964).

3 Böttiger M. The elimination of polio in the Scandinavian countries. Public Health Rev. 21(1-2), 27-33 (1993).

4 Chin TD, Marine WM, Hall EC et al. Poliomyelitis in Des Moines, Iowa, 1959. The influence of Salk vaccination on the epidemic pattern and the spread of the virus in the community. Am. J. Hyg. 74, 67-94 (1961).

5 Marine WM, Chin TD, Gravelle CR. Limitation of fecal and pharyngeal poliovirus excretion in Salk-vaccinated children. A family study during a type 1 poliomyelitis epidemic. Am. J. Hyg. 76, 173-195 (1962).

6 Gelfand HM, Potash L, Leblanc DR et al. Intrafamilial and interfamilial spread of living vaccine strains of polioviruses. JAMA 170(17), 2039-2048 (1959).

7 Duintjer Tebbens RJ, Pallansch MA, Chumakov KM et al. Expert review on poliovirus immunity and transmission. Risk Anal. 33(4), 544-605 (2013).

8 Sutter RW, Kew OM, Cochi SL et al. Poliovirus vaccine - live. In: Vaccines. Plotkin SA, Orenstein WA, Offit PA (Eds). Saunders Elsevier, PA, USA, 98-645 (2013).

9 Ghendon YZ, Sanakoyeva II. Comparison of the resistance of the intestinal tract to poliomyelitis virus (Sabin's strains) in persons after naturally and experimentally acquired immunity. Acta Virol. 5, 265-273 (1961).

10 Benyesh-Melnick M, Melnick JL, Rawls WE et al. Studies of the immunogenicity, communicability and genetic stability of oral poliovaccine administered during the winter. Am. J. Epidemiol. 86(1), 112-136 (1967).

11 Chen RT, Hausinger S, Dajani AS et al. Seroprevalence of antibody against poliovirus in inner-city preschool children. JAMA 275(21), 1639-1645 (1996).

12 Sabin AB, Ramos-Alvarez M, AlvarezAmezquita $\mathrm{J}$ et al. Live, orally given poliovirus vaccine: effects of rapid mass immunization on population under conditions of massive enteric infection with other viruses. JAMA 173(14), 1521-1526 (1960).

13 Alexander LN, Seward JF, Santibanez TA et al. Vaccine policy changes and epidemiology of poliomyelitis in the United States. JAMA 292(14), 1696-1701 (2004).

14 Dane DS, Dick GWA, Briggs $M$ et al. Vaccination against poliomyelitis with live virus vaccines: 6 . Changes in Sabin type II oral vaccine virus after human passage. $B r$. Med. J. 2(5247), 259-265 (1961).

15 Duintjer Tebbens RJ, Pallansch MA, Kim $\mathrm{J}-\mathrm{H}$ et al. Review: oral poliovirus vaccine evolution and insights relevant to modeling the risks of circulating vaccine-derived polioviruses (cVDPVs). Risk Anal. 23(4), 680-702 (2013).

16 Burns C, Diop O, Sutter RW et al. Vaccinederived polioviruses. J. Infect. Dis. 210 (Suppl. 1), S283-S293 (2014).

- Provides an overview of circulating vaccine-derived poliovirus events and known immunodeficiency-associated vaccinederived poliovirus excretors to date.

17 Kew O, Morris-Glasgow V, Landaverde $\mathrm{M}$ et al. Outbreak of poliomyelitis in Hispaniola associated with circulating type 1 vaccine-derived poliovirus. Science 296(5566), 356-359 (2002).

18 World Health Organization. Global Polio Eradication Initiative - circulating vaccinederived poliovirus cases 2000-2015. (2015). www.polioeradication.org/

19 Li L, Ivanova O, Driss N et al. Poliovirus excretion among persons with primary immune deficiency disorders: Summary of a seven-country study series. J. Infect. Dis. 210(Suppl. 1), S368-S372 (2014).

20 Duintjer Tebbens RJ, Pallansch MA, Thompson KM. Modeling the prevalence of immunodeficiency-associated long-term vaccine-derived poliovirus excretors and the potential benefits of antiviral drugs. BMC Infect. Dis. 15, 379 (2015).

21 Estívariz CF, Watkins M, Handoko D et al. A large vaccine-derived poliovirus outbreak on Madura Island - Indonesia, 2005. J. Infect. Dis. 197(3), 347-354 (2008).

22 Thompson KM, Pallansch MA, Duintjer Tebbens RJ et al. Pre-eradication vaccine policy options for poliovirus infection and disease control. Risk Anal. 33(4), 516-543 (2013).

23 Patriarca PA, Wright PF, John TJ. Factors affecting the immunogenicity of oral poliovirus vaccine in developing countries: review. Rev. Infect. Dis. 13, 926-939 (1991).

24 Grassly NC, Fraser C, Wenger J et al. New strategies for the elimination of polio from India. Science 314(5802), 1150-1153 (2006).
25 World Health Assembly. Global eradication of poliomyelitis by the year 2000 (1988). www.who.int/ihr/polioresolution4128en.pdf

26 World Health Organization. Transmission of wild poliovirus type 2 - apparent global interruption. Wkly Epidemiol. Rec. 76, 95-97 (2001).

27 Kew OM, Cochi SL, Jafari HS et al. Possible eradication of wild poliovirus type 3 - worldwide, 2012. MMWR Morb. Mortal. Wkly Rep. 63(45), 1031-1033 (2014).

28 World Health Organization. Polio this week as of 10 August 2016 (2016). http://reliefweb.int/sites/reliefweb.int/files

29 Thompson KM, Duintjer Tebbens RJ, Pallansch MA et al. The risks, costs, and benefits of possible future global policies for managing polioviruses. Am. J. Public Health 98(7), 1322-1330 (2008).

30 World Health Assembly. Poliomyelitis (Resolution 68.3) (2015). http://apps.who.int/gb/ebwha/pdf_files/

31 Global Polio Eradication Initiative. One Step Close to Eradication: the Withdrawal of Type Two Oral Polio Vaccine Complete. www.polioeradication.org/mediaroom/

32 World Health Organization. Global Polio Eradication Initiative: Polio Eradication and Endgame Strategic Plan (2013-2018). www.polioeradication.org/resourcelibrary

33 Duintjer Tebbens RJ, Pallansch MA, Cochi SL et al. An economic analysis of poliovirus risk management policy options for 2013-2052. BMC Infect. Dis. 15, 389 (2015).

- Describes an integrated global model for long-term poliovirus risk management and estimates health and economic outcomes of main policy options.

34 Thompson KM, Duintjer Tebbens RJ. National choices related to inactivated poliovirus vaccine, innovation, and the end game of global polio eradication. Exp. Rev. Vaccines 13(2), 221-234 (2014).

35 Bandyopadhyay AS, Garon J, Seib K et al. Polio vaccination: past, present and future. Future Microbiol. 10(5), 791-808 (2015).

-. Highlights polio endgame issues and reviews current state of research into new poliovirus vaccines.

36 Okayasu H, Sutter RW, Jafari HS et al. Affordable inactivated poliovirus vaccine: strategies and progress. J. Infect. Dis. 210(Suppl. 1), S459-S464 (2014).

37 Anis E, Kopel E, Singer S et al. Insidious reintroduction of wild poliovirus into Israel, 2013. Euro Surveill. 18(38) pii=20586 (2013). 
- Describes episode of widespread serotype 1 wild poliovirus transmission despite very high inactivated poliovirus vaccine coverage, highlighting the ability of inactivated poliovirus vaccine recipients to participate asymptomatically in poliovirus transmission.

38 Kalkowska DA, Duintjer Tebbens RJ, Grotto I et al. Modeling options to manage type 1 wild poliovirus imported into Israel in 2013. J. Infect. Dis. 211(11), 1800-1812 (2015).

39 Garon J, Seib K, Orenstein WA et al. Polio endgame: the global switch from tOPV to bOPV. Exp. Rev. Vaccines 15(6), 693-708 (2016).

40 Minor PD. An introduction to poliovirus: pathogenesis, vaccination, and the endgame for global eradication. Methods Mol. Biol. 1387, 1-10 (2016).

41 Patel M, Zipursky S, Orenstein W et al. Polio endgame: the global introduction of inactivated polio vaccine. Exp. Rev. Vaccines 14(5), 749-762 (2015).

42 Parker EP, Molodecky NA, Pons-Salort $\mathrm{M}$ et al. Impact of inactivated poliovirus vaccine on mucosal immunity: implications for the polio eradication endgame. Exp. Rev. Vaccines 14(8), 1113-1123 (2015).

43 Minor P. The polio endgame. Hum. Vaccin. Immunother. 10(7), 2106-2108 (2014).

44 Sutter RW, Platt L, Mach O et al. The new polio eradication end game: rationale and supporting evidence. J. Infect. Dis. 210 (Suppl. 1), S434-S438 (2014).

45 Grassly NC. The final stages of the global eradication of poliomyelitis. Philos. Trans. $R$. Soc. Lond. B Biol. Sci. 368(1623), 20120140 (2013).

46 Estivariz CF, Pallansch MA, Anand A et al. Poliovirus vaccination options for achieving eradication and securing the endgame. Curr. Opin. Virol. 3(3), 309-315 (2013).

47 Davis R, Wright PF. Circulating vaccine derived poliovirus and the polio eradication endgame. Pan Afr. Med. J. 12, 109 (2012).

48 Thompson KM, Duintjer Tebbens RJ. Current polio global eradication and control policy options: perspectives from modeling and prerequisites for OPV cessation. Expert Rev. Vaccines 11(4), 449-459 (2012).

49 Aylward B, Yamada T. The polio endgame. $N$. Engl. J. Med. 364(24), 2273-2275 (2011).

50 Modlin J, Wenger J. Achieving and maintaining polio eradication - new strategies. N. Engl. J. Med. 371(16), 1476-1479 (2014).
51 Duintjer Tebbens RJ, Pallansch MA, Kew OM et al. Risks of paralytic disease due to wild or vaccine-derived poliovirus after eradication. Risk Anal. 26(6), 1471-1505 (2006).

52 Thompson KM, Duintjer Tebbens RJ. Modeling the dynamics of oral poliovirus vaccine cessation. J. Infect. Dis. 210 (Suppl. 1), S475-S484 (2014).

53 Duintjer Tebbens RJ, Thompson KM. Modeling the potential role of inactivated poliovirus vaccine to manage the risks of oral poliovirus vaccine cessation. J. Infect. Dis. 210(Suppl. 1), S485-S497 (2014).

54 Duintjer Tebbens RJ, Pallansch MA, Wassilak SGF et al. Characterization of outbreak response strategies and potential vaccine stockpile needs for the polio endgame. BMC Infect. Dis. 16, 137 (2016).

-• Modeling analysis of different outbreak response strategies that suggests the ability of rapid monovalent oral poliovirus vaccine (OPV) outbreak response to control most outbreaks during the first 5 years after OPV cessation, but also demonstrates challenges associated with current vaccines to control outbreaks more than 5 years after OPV cessation.

55 Duintjer Tebbens RJ, Pallansch MA, Wassilak SGF et al. Combinations of quality and frequency of immunization activities to stop and prevent poliovirus transmission in the high-risk area of northwest Nigeria. PLoS ONE 10(6), e0130123 (2015).

56 Thompson KM, Duintjer Tebbens RJ. The differential impact of oral poliovirus vaccine formulation choices on serotype-specific population immunity to poliovirus transmission. BMC Infect. Dis. 15, 374 (2015).

57 Jafari H, Deshpande JM, Sutter RW et al. Polio eradication. Efficacy of inactivated poliovirus vaccine in India. Science 345(6199), 922-925 (2014).

58 John J, Giri S, Karthikeyan AS et al. Effect of a single inactivated poliovirus vaccine dose on intestinal immunity against poliovirus in children previously given oral vaccine: an open-label, randomised controlled trial. Lancet 384(9953), 1505-1512 (2014).

59 World Health Organization. GAP III: WHO Global Action Plan to minimize poliovirus facility-associated risk after type-specific eradication of wild polioviruses and sequential cessation of oral polio vaccine use (2015).

www.polioeradication.org/Portals

60 World Health Organization. New polio vaccines for the post-eradication era. Report number: WHO/V\&B/00.20 (2000) www.who.int/biologicals/publications/

61 Heymann DL, Sutter RW, Aylward RB. A global call for new polio vaccines. Nature 434(7034), 699-700 (2005).

62 Ehrenfeld E, Modlin J, Chumakov K. Future of polio vaccines. Expert Rev. Vaccines 8(7), 899-905 (2009).

63 Chumakov K, Ehrenfeld E. New generation of inactivated poliovirus vaccines for universal immunization after eradication of poliomyelitis. Clin. Infect. Dis. 47(12), 1587-1592 (2008).

64 Macadam AJ, Ferguson G, Stone DM et al. Rational design of genetically stable, live-attenuated poliovirus vaccines of all three serotypes: relevance to poliomyelitis eradication. J. Virol. 80(17), 8653-8663 (2006).

65 Lauring AS, Jones JO, Andino R. Rationalizing the development of live attenuated virus vaccines. Nat. Biotechnol. 28(6), 573-579 (2010).

66 Sanders BP, De Los Rios Oakes I, Van Hoek $\mathrm{V}$ et al. Cold-adapted viral attenuation (CAVA): highly temperature sensitive polioviruses as novel vaccine strains for a next generation inactivated poliovirus vaccine. PLoS Pathog. 12(3), e1005483 (2016).

67 Knowlson S, Burlison J, Giles E et al. New strains intended for the production of inactivated polio vaccine at low-containment after eradication. PLoS Pathog. 11(12), e1005316 (2015).

68 Norton EB, Bauer DL, Weldon WC et al. The novel adjuvant $\mathrm{dmLT}$ promotes dose sparing, mucosal immunity and longevity of antibody responses to the inactivated polio vaccine in a murine model. Vaccine 33(16), 1909-1915 (2015).

69 Saez-Llorens X, Clemens R, Leroux-Roels G et al. Immunogenicity and safety of a novel monovalent high-dose inactivated poliovirus type 2 vaccine in infants: a comparative, observer-blind, randomised, controlled trial. Lancet Infect. Dis. 16(3), 321-330 (2016).

70 World Health Organization. WHO guide for standardization of economic evaluations of immunization programmes. Report number: WHO/IVB/0814 (2008). http://apps.who.int/iris/bitstream/

71 Gold MR, Siegel JE, Russel LB et al. Cost-Effectiveness In Health and Medicine. Oxford University Press, NY, USA (1996).

72 Duintjer Tebbens RJ, Pallansch MA, Kalkowska DA et al. Characterizing poliovirus transmission and evolution: insights from modeling experiences with wild 
and vaccine-related polioviruses. Risk Anal. 23(4), 703-749 (2013).

73 Duintjer Tebbens RJ, Sangrujee N, Thompson KM. The costs of polio risk management policies after eradication. Risk Anal. 26(6), 1507-1531 (2006).

74 World Bank. World Bank list of economies (2012).

http://siteresources.worldbank.org/

75 Duintjer Tebbens RJ, Thompson KM. Managing the risk of circulating vaccinederived poliovirus during the endgame: oral poliovirus vaccine needs. BMC Infect. Dis. 15 , 390 (2015).
76 Duintjer Tebbens RJ, Thompson KM. Comprehensive screening for immunodeficiency-associated vaccine-derived poliovirus: an essential OPV cessation risk management strategy (in preparation). Epidemiol. Infect. doi:10.1017/ S0950268816002302 (2016) (Epub ahead of print).

77 Duintjer Tebbens RJ, Thompson KM. Uncertainty and sensitivity analysis of cost assumptions for global long-term poliovirus risk management (in preparation). J. Vaccines Vaccin. 7(5), 339 (2016).

78 Plotkin SA, Mahmoud AA, Farrar J. Establishing a global vaccine-development fund. N. Engl. J. Med. 373(4), 297-300 (2015).

79 Duintjer Tebbens RJ, Hampton LM, Thompson KM. Implementation of coordinated global serotype 2 oral poliovirus vaccine cessation: risks of potential non-synchronous cessation. BMC Infect. Dis. 16, 237 (2016).

80 Duintjer Tebbens RJ, Kalkowska DA, Wassilak SGF et al. The potential impact of expanding target age groups for polio immunization campaigns. BMC Infect. Dis. 14, 45 (2014). 